\title{
Separability of n-particle mixed states: necessary and sufficient conditions in terms of linear maps
}

\author{
Michał Horodecki \\ Departament of Mathematics and Physics \\ University of Gdańsk, 80-952 Gdańsk, Poland \\ Paweł Horodecki \\ Faculty of Applied Physics and Mathematics \\ Technical University of Gdańsk, 80-952 Gdańsk, Poland \\ Ryszard Horodecki* \\ Institute of Theoretical Physics and Astrophysics \\ University of Gdańsk, 80-952 Gdańsk, Poland
}

\begin{abstract}
We provide necessary and sufficient conditions for separability of mixed states of n-particle systems. The conditions are formulated in terms of maps which are positive on product states of $n-1$ particles. The method of providing of the maps on the basis of unextendable product bases is provided. The three qubit state problem is reformulated in the form suggesting possibility of explicite characterisation of all maps needed for separability condition.
\end{abstract}

\section{INTRODUCTION}

Quantum entanglement [1.2] is one of the most intriguing quntum phenomena leading to various quantum effects with possible realisations in quantum theory. The main problem is to find necessary and sufficient conditions for existence of entanglement of noisy quantum state. It can be formulated as a problem of serching necessary and sufficient conditions for separability of the state or physically speaking - for nonexistence of quantum correlations in the system state. The problem is nontrivial even for bipartite case i. e. the case of quantum system consiting of two subsystems. The practical, operational solution of the problem in bipartite case is known only for the simplest $2 \otimes 2$, $2 \otimes 3$ case where the positivity of so called partial transposition has been shown to be necessary and sufficient condition for separability [3, 4 ]. For higher dimensional bipartite system it is not the case [4 6]. The general characterisation of separabilty for those case is given in term of so called positive maps PM which, however, have not been operationally characterised so far. Recently the progress in this direciton has been made [7,8] which suggests that positive maps tests of separability will have more and more practical applications in reasonable feature.

In quantum information theory instead of bipartite case we deal, in general, with multipartite one like in quantum computing (see [12]). Still there is a problem with the noise as far as practical implementation of quantum computing is concerned.

The definition of multiparticle entanglement and separability was introduced in 10,11 following the one for bipartite case [9]. The it was considered in case of distillability of multiparticel entanglement [13, 14], separability of very noisy mixed states [15,16] and its implications fo NMR quantum computing [17 19]. It was also analysed form the point of veiw of invariants under local fransformations [20,21] and their applications for quantum cryptography [22]. The very interesting aspects of multiparticle mixed states entanglement were studied in detailes in context distillation and of bound entanglement phenomena [23 26], quantum telecloning [27], fragility of entanglement [28] as well as quantum to classical phase transitions in quantum computers [29].

So it is important to develop the formalism which could give general tools for testing of presence (or absence) of multiparticle entanglement in given state system. In this paper we shall make step in this direciton firmulationg the necessary and sufficient condition of separability of (in general mixed) multiparticle states in terms of new kind of linear maps i. e. linear maps positive on product states (LMPP). We shall also provede the construciton of such maps basing on UPB 23:24 method of generation of bound entanglememt as well as the technique provieded in

*e-mail: fizrh@univ.gda.pl 
[31]. Finally we shall discuss in more detailes the three qubit case showing that one can hope that some operational characterisation of LMPP, (and - equivalently - of the corresponding entanglement witnesess) is possible.

\section{REPETITION: CHARACTERISATION OF BIPARTITE SEPARABILITY - TWO DUAL APPROACHES}

Below we shall briefly racall the way of proving the mentioned result as well as the positive maps characterisation of separability stressing some special aspect of the question.

Let us recall briefly the definition of the bipartite separability in finitedimensional case.

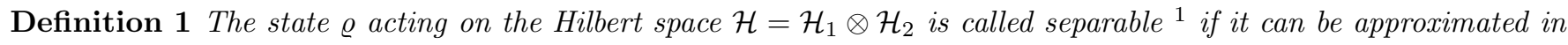
the trace norm by the states of the form

$$
\varrho=\sum_{i=1}^{k} p_{i} \varrho_{i} \otimes \tilde{\varrho}_{i}
$$

where $\varrho_{i}$ and $\tilde{\varrho}_{i}$ are states on $\mathcal{H}_{1}$ and $\mathcal{H}_{2}$ respectively.

Usually we deal with a finite dimensional Hilbert space $\operatorname{dim} \mathcal{H}=N<\infty$. For this case it is known [5] that any separable state can be written as a convex combination of finite product pure states $k \leq N^{2}$, i.e. in those cases the "approximation" part of the definition is redundant.

Now separability can be characterised in two equivalent, though different ways 值.

First we have characterisation in terms of operators (called, following [31], "entanglement witnesses") negative mean values of which are indicators of entanglement. We have the flowing definition (cf. 31):

Definition 2 The observable $W$ is called entanglement witness iff its mean values on all separable states are nonnegative i. e. iff $\operatorname{Tr}(\varrho W) \geq 0$ for all separabile $\varrho$.

Now we have the following characterisation of separability [4]:

Characterisation (I) $\varrho$ is separable iff $\operatorname{Tr}(\varrho W) \geq 0$ for all entanglement witnesses $W$.

On the other hand we have the characterisation in terms of positive maps which are defined as follows (see [33, 32]):

Definition 3 Let $\mathcal{B}(\mathcal{H})$ stand for the algebra of all operators on Hilbert space $\mathcal{H}$, $\operatorname{dim} \mathcal{H}<\infty$. The the map $\Lambda$ : $\mathcal{B}\left(\mathcal{H}_{2}\right) \rightarrow \mathcal{B}\left(\mathcal{H}_{1}\right)$ is positive iff $\Lambda(A) \geq 0$ for all $A \geq 0, A \in \mathcal{B}\left(\mathcal{H}_{1}\right)$.

Recall that $X \geq 0$ stands for hermitian operator with nonegative eigenvalues and such operator is called positive.

Now we can recall the second characterisation of separability in terms of positive maps

Characterisation (II) 四 $\varrho$ defined on $\mathcal{H}_{1} \otimes \mathcal{H}_{2}$ is separable iff operator $I \otimes \Lambda(\varrho) \geq 0$ for all positive $\Lambda$.

Remark .- The above characterisation relies on the fact that there are the positive maps $\Lambda$ such that their multiplication by identity $I \otimes \Lambda$ is not positive. The well known map ot that kind is simply transposition $T$ [32,33] defined on martix elements as $[T(A)]_{m n}=[A]_{n m}$. The map $I \otimes T$ is not positive and the condition $I \otimes T(\varrho) \geq 0$ is the form of separability criterion introduced by Peres [3].

What is the difference betweeen the characteriastions (I) and (II) ? The first has a scalar character as it utilises conditions imposed on mean values, while the second one applies the operator conditions imposing the boundaries on the spectrum of some operators.

Being both characterisation of the same set the conditions (I) and (II) are both equivalent if considered collectively. The link is fomed by the fact that there is one to one isomorphism between positive maps and entanglement witnesses A coming form Ref. [32]. Namely

$$
A \leftrightarrow I \otimes \Lambda_{A}\left(P_{+}\right)
$$

Where $P_{+}=|\Psi\rangle\langle\Psi|,| \Psi\rangle=\sum_{i=1}^{\operatorname{dim} \mathcal{H}_{1}}|k\rangle \otimes|k\rangle,|\Psi\rangle \in \mathcal{H}_{1} \otimes \mathcal{H}_{1}$. What is the motivation to look rather positive maps then entanglement witnesses? Though characterisations (I) and (II) are completely equivalent the single conditions

\footnotetext{
${ }^{1}$ The present definition is due to Werner [9], who called them classically correlated states.
} 
(i) $\operatorname{Tr}(\varrho A) \geq 0$ (ii) $I \otimes \Lambda_{A}(A) \geq 0$ are not. In particular sometimes the second one is much stronger than the first one. Let us recall the $2 \otimes 2$ example corresponding to the system composed of two $\frac{1}{2}$-spin systems.

Example. Let $A=V$ where $V$ is a "flip" operator i. e. $V|\phi\rangle \otimes|\psi\rangle=|\psi\rangle \otimes|\phi\rangle$. It has been shown [9] that $V$ is a legitimite entanglement witness i. e. it gives nonegative mean value on product states, ergo has the same property on all separable states. On the other hand it detects entanglement of all states $\sigma$ with property $\left\langle\Psi_{-}|\sigma| \Psi_{-}\right\rangle>\frac{1}{2}$, $\left|\Psi_{-}\right\rangle=\frac{1}{\sqrt{2}}(|01\rangle-|10\rangle)$ Hence $\operatorname{Tr}(A \varrho) \geq 0$ is nontrivial separability condition. However it is weak condition - it does not detect for example entanglement of maximally entangled state $\frac{1}{\sqrt{2}}(|00\rangle+|11\rangle)$ giving on it strictly positive value 1 .

But let us apply isomorphism (2). Then we get the map $V \rightarrow \Lambda_{V}=T$ which is the transposition. But it was shown [4] that $I \otimes T(\varrho)=I \otimes \Lambda_{V}(\varrho) \geq 0$ is sufficient condition for separability of mixed states hence it detect all entanglement. Thus in this case single condition from Characteristion (II) appeared to be much more powerfull than form (I). In this way we have seen the main motivation to study the problem maps dececting entanglement in place of entanglement witnesses.

Finally we recall that there are two other weaker notions introduced in context of separability. Namely we have the definition (see [23]):

Definition 4 The state of n-partite system is called semiseparable iff for any index $m(1 \leq m \leq n)$ the state can be represented as a separable state of bipartite system composed form $m$-th subsystem and the rest of the system:

$$
\varrho=\sum_{i=1}^{N} p_{i} \varrho_{m}^{i} \otimes \varrho_{1, \ldots, m-1, m+1, \ldots n}^{i} .
$$

It has been shown that there are semiseparable states whic are not separable 23,24]. The characterisations I, II recalled above can be easily applied to give necessary and sufficient conditions of semiseparability. It is enough to divide system into proper subsystems and apply the condition, then divide into two other ones and apply the corresponding separability condition again, etc.

Finally there is another definition concerning very interesting property. Namely one can imagine that we have entanglement in the system composed form $n$ elementary subsystems but it is represented by "clusters" of some restricted size (in number of elementary subsystems involved). We have the corresponding definition [1]:

Definition 5 State @ does not represent entanglement of not more than $k$ elementary subsystems iff it admits decomposition

$$
\varrho=\sum_{i=1}^{N} p_{i} \varrho^{i, 1} \otimes \ldots \otimes \varrho^{i, n_{i}}
$$

and $\varrho^{i, n_{i}}$ describes subset of not more than $k$ subsystems form all $n$ elementary ones.

We discuss the above notion in the context of the present results.

\section{NECESSARY AND SUFFICIENT CONDITIONS FOR SEPARABILITY OF N-PARTICLE SYSTEMS}

The definition of separable states [9] was easily generalized to systems composed of more than two subsystems (see for example [34]):

Definition 6 The state @ acting on the Hilbert space $\mathcal{H}=\underset{l=1}{\otimes} \mathcal{H}_{l}$ is called separable if it can be approximated in the trace norm by the states of the form

$$
\varrho=\sum_{i=1}^{k} p_{i} \underset{l=1}{\otimes} \varrho_{i}^{l}
$$

where $\varrho_{i}^{l}$ are states on $\mathcal{H}_{l}$.

Straightforward generalization of the proof about decomposition from Ref. [5] gives us the possibility of omitting the approximation part in the definition: 
Lemma 1 Any separable state @ of a system composed by $m$ subsystems can be written as:

$$
\varrho=\sum_{i=1}^{k} p_{i} P_{\text {prod }}^{i}, k \leq N^{2}
$$

where $P_{\text {prod }}^{i}$ are pure product states having the $n$-decomposable form $\underset{l=1}{\otimes} P_{l}$, where $P_{l}$ are projectors acting on $\mathcal{H}_{l}$.

Now following the proof form the Ref. 四 can provide the simple generalisation of the Characterisation (I) form the previous section.

Theorem 1 State $\varrho$ defined on $\mathcal{H}=\mathcal{H}_{1} \otimes \mathcal{H}_{2} \otimes \ldots \mathcal{H}_{n}$ is separable iff $\operatorname{Tr}(A \varrho) \geq 0$ holds for all observables $A$ on $\mathcal{H}$ such that

$$
\operatorname{Tr}\left(A P_{1} \otimes P_{2} \otimes \ldots \otimes P_{n}\right) \geq 0
$$

for all product projections $P_{1} \otimes P_{2} \otimes \ldots \otimes P_{n}$ ( $P_{i}$ are projectors on $\left.\mathcal{H}_{i}\right)$.

Remark.- As the conclusion from the Hahn-Banach theorem is valid for any Banach space our theorem can be generalized for infinitely dimensional Hilbert spaces.

Now we provide the following theorem which is nontrivial generalisation of Characterisation (II) to the multiparticle case.

Theorem 2 State $\varrho$ defined on $\mathcal{H}=\mathcal{H}_{1} \otimes \mathcal{H}_{2} \otimes \ldots \mathcal{H}_{n}$ is separable iff $I \otimes \tilde{\Lambda}(\varrho) \geq 0$ for all LMPP $\tilde{\Lambda}$ i. e. all linear maps $\tilde{\Lambda}: \mathcal{B}\left(\mathcal{H}_{2} \otimes \ldots \mathcal{H}_{n}\right) \rightarrow \mathcal{B}\left(\mathcal{H}_{1}\right)$ such that

$$
\tilde{\Lambda}\left(P_{2} \otimes \ldots \otimes P_{n}\right) \geq 0
$$

for all product projections $P_{2} \otimes \ldots \otimes P_{n}$ ( $P_{i}$ are projectors on $\left.\mathcal{H}_{i}\right)$.

Proof .- We shall prove only the "if" part as the only if is trivial. We shall show that iff the condition (8) holds for all required $\tilde{\Lambda}$ than (7) takes place for all entanglement witnesses $A$. Let (\$) hold for all maps $\tilde{\Lambda}$. Than in particular $\operatorname{Tr}\left(P_{+} \tilde{\Lambda}(\varrho)\right) \geq 0$ or $\operatorname{Tr}\left(I \otimes(\tilde{\Lambda})^{\dagger}\left(P_{+}\right) \varrho\right) \geq 0$. Hence the assumption (\&) leads to the condition $\operatorname{Tr}(\tilde{A} \varrho) \geq 0$ for

$$
\tilde{A}=I \otimes(\tilde{\Lambda})^{\dagger}\left(P_{+}\right) .
$$

Now it is enough to show that any entanglement witness is of the above form for some $\Lambda^{\dagger}$ such that $I \otimes \Lambda^{\dagger}\left(P_{2} \otimes \ldots \otimes P_{n}\right) \geq$ 0 for all $P_{i}$ being projections on $\mathcal{H}_{i}$. Take any entanglement witness $A$. Then it must satisfy (7) and on the other hand it must be of the form (2) for some map $\Lambda: \mathcal{B}\left(\mathcal{H}_{1}\right) \rightarrow \mathcal{B}\left(\mathcal{H}_{2} \otimes \ldots \otimes \mathcal{H}_{n}\right)$. P Let design by $\Lambda_{1}=\Lambda^{\dagger}$. Then the condition (『) is equivalent to the form

$$
\operatorname{Tr}\left(I \otimes \Lambda_{1}^{\dagger}\left(P_{+}\right) P_{1} \otimes P_{2} \otimes \ldots \otimes P_{n}\right)=\operatorname{Tr}\left(P_{+} P_{1} \otimes \Lambda_{1}\left(P_{2} \otimes \ldots \otimes P_{n}\right)\right) \geq 0
$$

Now let us recall that (i) $P_{+}^{T_{1}}=V$ where $V$ is a flip operator acting on $\mathcal{H}_{1} \otimes \mathcal{H}_{1}$ (ii) $\operatorname{Tr}(X Y)=\operatorname{Tr}\left(X^{T_{1}} Y^{T_{1}}\right)$, (iii) [9] $\operatorname{Tr}(V X \otimes Y)=\operatorname{Tr}\left(X^{T_{1}} Y\right)$. Application of those properties leads us to the conclusion that the above equation is equivalent to the one

$$
\operatorname{Tr}\left(P_{1} \Lambda_{1}\left(P_{2} \otimes \ldots \otimes P_{n}\right)\right) \geq 0
$$

Form arbitrariness of projectors $P_{i}$ we get immediately that $\Lambda_{1}$ posess the property of $\tilde{\Lambda}$ maps form the theorem (2). Hence any entanglement witness is of the form 9 which ends the proof.

\footnotetext{
${ }^{2}$ The latter comes form the fact that (2) gives general isomorphism between linear maps and hermitian operators.

${ }^{3}$ Here $X^{T_{1}} \equiv T \otimes I(X)$ i. e. partial transposition with respect to the first Hilbert space $\mathcal{H}_{1}$.
} 


\section{MULTIPARTICLE CASE - CONSTRUCTION BASED ON UNEXTENDIBLE PRODUCT BASES}

In this section we shall utilise a pioneering method of Ref. [31] to produce maps positive on product states of two q-bits. The method was the following producing the positive maps which are indecomposable. Namely it has been shown [23,24] that there exist so called unextendable product bases (UPB) i. e. such sets of product vectors in $\mathcal{H}=\mathcal{H}_{1} \otimes \mathcal{H}_{2} \otimes \ldots \otimes \mathcal{H}_{n}$ that (i) there os no product vector orthogonal to them (ii) they do not span the whole space $\mathcal{H}$. It produced systematic way of generating so called bound entangled states i. e. entangled states form which no entanglement can be distilled. The systematis method is as follows: find UPB set then take the projector $P_{U P B}^{\perp}$ onto space orthogonal to the set and normalise it. The resulting state $\varrho_{U P B}$ is bound entangled. In particular it has PPT property with respect to any division of the system onto two parts. For bipartite $\varrho_{U P B}$ their structure was utilised to get indecomposable maps in the following way [31]: take the projector onto the UPB set $P_{U P B}$ of bipartite states, calculate minimal value of $\epsilon=\max _{P \otimes Q} \operatorname{Tr}\left(P_{U P B} P \otimes Q\right)$. Then take maximally entangled state $\Psi_{+}$and calculate maximal value $c=\max _{P \otimes Q} \operatorname{Tr}\left(P_{U P B} P \otimes Q\right)$ which is strictly positive. Finally the following operator

$$
A=P_{U P B}-\frac{\epsilon}{c} P_{+}
$$

is an entanglement witness for $\varrho_{U P B}$ i. e. it is has positive mean value on all product states but negative on $\varrho_{U P B}$. The corresponding linear map generated automatically via isomorphism (2) is positive indecomposable map (see [31] for details).

Below we shall show that the same way can lead to production of positive maps on products if applied to multipartite systems 1 . Consider any multipartite system and its bound entangled state $\varrho_{U P B}$ produced by given UPB set. Then take the following operator

$$
\tilde{A} \equiv P_{S_{U P B}}-\frac{\epsilon}{c} C
$$

where $C \geq 0$ is an arbitrary positive operator with $\operatorname{Tr}\left(P_{S_{U P B}}^{\perp} C\right) \geq 0$ and $c=\underset{P_{1} \otimes \ldots \otimes P_{N}}{\max } \operatorname{Tr}\left(C P_{1} \otimes \ldots \otimes P_{N}\right)$. Then the operator $\tilde{A} \in \mathcal{B}\left(\mathcal{H}_{1} \otimes \ldots \otimes H_{N}\right)$ has by the very definition positive mean values on product states. So it satisfies conditions of the Theorem 2. On the other hand it has negative mean value on $\varrho_{U P B}$ :

$$
\operatorname{Tr}\left(\tilde{A} \varrho_{U P B}\right)=-\frac{\epsilon}{c}<0
$$

being then entanglement witness for this state. By the theorem that the corresponding linear map $\Lambda_{\tilde{A}}$ (this time via relation (9) is postive on product states. On the other hand it detects multipartite entanglement as $\left\langle\Psi_{+}\right| I_{1} \otimes$ $\Lambda_{\tilde{A}} \varrho_{U P B}\left|\Psi_{+}\right\rangle=\operatorname{Tr}\left(\tilde{A} \varrho_{U P B}\right)<0$. The above construction if applied of the states generated by the most simple three qubits UPB [23] $S_{U P B}=\operatorname{span}\{|000\rangle,|+1-\rangle,|1-+\rangle,|+-1\rangle\}$. (with $| \pm\rangle=\frac{1}{\sqrt{2}}(|0\rangle \pm|1\rangle)$ ) leads to the map which is, however, of complicated form. Below we shall analyse in more detailes the properties of maps revealing entanglement of three qubits.

\section{THREE Q-BIT CASE - GENERAL ANALYSIS}

Consider now again the case of three q-bits i. e. the system defined on a Hilbert space $\mathcal{H}=\mathcal{H}_{1} \otimes \mathcal{H}_{2} \otimes \mathcal{H}_{2}=$ $\mathcal{C}^{2} \otimes \mathcal{C}^{2} \otimes \mathcal{C}^{2}$. We have the following obesrvation:

Observation .- Any entanglement witness (8) $\tilde{\Lambda}: \mathcal{B}\left(\mathcal{C}^{2} \otimes \mathcal{C}^{2}\right) \rightarrow \mathcal{B}\left(\mathcal{C}^{2}\right)$ satisfies $\forall \sigma_{a}, \sigma_{b}, \sigma_{c}\left(\sigma_{x}\right.$ - one q-bit states)

$$
\operatorname{Tr}\left[\sigma_{a} \tilde{\Lambda}\left(\sigma_{b} \otimes \sigma_{c}\right)\right] \geq 0
$$

or, equivalently,

$$
\operatorname{Tr}\left[\tilde{\Lambda}^{\dagger}\left(\sigma_{a}\right) \sigma_{b} \otimes \sigma_{c}\right] \geq 0
$$

Now we have the following theorems:

\footnotetext{
${ }^{4}$ The first part of reasoning presented below (concerning entanglement witnesses) was independently performed by B. Terhal [35]
} 
Theorem 3 The three $q$-bit state $\varrho$ is separable iff $I \otimes \tilde{\Lambda}(\varrho) \geq 0$ for all $\tilde{\Lambda}: \mathcal{B}\left(\mathcal{C}^{2} \otimes \mathcal{C}^{2}\right) \rightarrow \mathcal{B}\left(\mathcal{C}^{2}\right)$ satisfies $\forall \sigma$ ( $\sigma$ one qubit state)

$$
\tilde{\Lambda}(\sigma)=A+B^{T_{2}}
$$

for some positive $A$ and $B$.

Below we shall write down the problem solution of which would be sufficient for characterisation of tripartite entanglement. Namely all the maps we need are the maps acting in the following way

$$
\tilde{\Lambda}[(I \otimes \hat{n} \vec{\sigma}) \otimes(I \otimes \hat{m} \vec{\sigma})]=\alpha(\hat{n}, \hat{m})[I+\vec{k}(\hat{n}, \hat{m}) \vec{\sigma}]
$$

where $\hat{x} \vec{\sigma} \equiv \sum_{i=1}^{3}, x_{i} \sigma_{i}$ where $\left\{x_{i}\right\}$ represent coordinates of real unit vector $\hat{x}$, while $\sigma_{i}$ stands for Pauli matrices. The vector $\vec{k}$ must satisfy $\|\vec{k}\| \leq 1$ and $\alpha \geq 0$. It is not easy to see whether it is possible to characterise the maps of that form. Nevertheless this is the first time when aiming in characterisation of the map with respect to somehow positivity we have clear positivity conditions - via treedimensional Bloch vectors) - on both its arguments and the results. It follows from the fact that triparticle separability condition for $2 \otimes 2 \otimes 2$ system is more restrictive then separability of $2 \otimes 4$ system. Actually there is more maps of that kind. This can be simply argued from the fact that three q-bits semiseparability (i.e. separability of state on $\mathcal{C}^{2} \otimes \mathcal{C}^{2} \otimes \mathcal{C}^{2}$ with respect to all "cuts" into two parts of type $\mathcal{C}^{2} \otimes \mathcal{C}^{4}$ ) is not equivalent to separability [23] ( we shall use this fact in the next section). As there is more maps positive on product states then the positive ones it may happen that the set of LMPP, strictly larger than the set of $\mathrm{PM}$, is also more regular and possible to characterise analytically.

\section{DISCUSSION}

We have provided the necessary and sufficient conditions of separability of multiparticle quantum systems in terms of maps positive on product states. This is a new class of maps forming a subset of set of positive maps. The construciton of examples basing on existence of unextendable product bases as well as the corresponding bound entangled states has been provided. For the case of three q-bits the necessary and sufficient condition for maps of the form can be formulated using the Bloch vector formalism which allow to hope that some operational charaterisation of the of the set of the maps is possible.

Let us outline the problem for further research in the context of the present results. As we have mentioned, the previous results can be easily applied to characterise semiseparability. The present ones can be applied to verions of partial semiseparability i. e. when systems is divided into, for example, three parts and is to be separable with respect to such partition). there is however an open problem how to apply it for the third definition (involving "clusters" of size $k$, see section II). In fact, the naive application of the present criteria should, in principle fail. Indeed, the simple inspection shows that it can happen that clusters can be shifted. Due to this fact, if applying the present approach naively, one can have fake detection of entanglement of, say, $k+1$ subsystems while only $k$-size entanglement is present. So there is need for further investigation towards characterisation of separability in sense of definition 3 .

In the present paper we have an example of case when physical problem - characterisation of multiparticle separability (or - equivalently - entanglement) leads to new mathematical objects linear maps positive on products investigation structure of which can be interesting itself. Moreover we know that, in general, single maps condition is much more powerfull that the entanglement witness condition so after finding entanglement witness for multiparticle case explicite generation of linear maps positive on product states (LMPP) may give much stronger separability conditions too which is of practical importance.

[1] A. Einstein, B. Podolsky and N. Rosen, Phys. Rev. 47 (1935) 777.

[2] E. Schrödinger, Naturwissenschaften, 23 (1935) 807.

[3] A. Peres, Phys. Rev. Lett. 77, 1413 (1996).

[4] M. Horodecki, P. Horodecki and R. Horodecki, Phys. Lett. A223, 8 (1996).

[5] P. Horodecki, Phys. Lett. A 232, 333 (1997).

[6] M. D. Choi, Proc. Sympos. Pure Math. 38, 583 (1982). 
[7] M. Lewenstein, B. Kraus, J. I. Cirac, and P. Horodecki, quant-ph/0005014.

[8] M. Lewenstein, B. Kraus, P. Horodecki, and J. I. Cirac. quant-ph/0005112.

[9] R. F. Werner, Phys. Rev. A 40 (1989) 4277.

[10] V. Vedral, M. B. Plenio, M. A. Rippin and P. L. Knight, Phys. Rev. Lett. 78 (1997) 2275.

[11] V. Vedral, M. B. Plenio, K. Jacobs and P. L. Knight, Phys. Rev. A 58 (1998) 883.

[12] A. Steane, Rep. Prog. Phys. 61 (1998) 117.

[13] M. Murao, M. B. Plenio, S. Popescu, V. Vedral and P. L. Knight, Phys. Rev. A, 57 (1998) R4075.

[14] W. Dür, J.I. Cirac and R. Tarrach, Phys. Rev. Lett. 83 (1999) 3562.

[15] K. Życzkowski, Phys. Rev. A 58 (1998) 883.

[16] G. Vidal and R. Tarrach Phys. Rev. A 59 (1999) 141

[17] S. Braunstein, C. M. Caves, R. Jozsa, N. Lindena and S. Popescu, Phys. Rev. Lett. 83 (1999) 1054.

[18] R. Schack and C. Caves, Phys. Rev. A 60 (1999) 4354.

[19] N. Linden and S. Popescu quant-ph/9906008.

[20] N. Linden and S. Popescu Forschr. Phys. 49 (1998) 567.

[21] N. Linden, S. Popescu and A. Sudbery, Phys. Rev. Lett. 83 (1999) 243.

[22] J. Kempe, Phys. Rev. A 60 (1999) 910.

[23] C. H. Bennett, D. P. DiVincenzo, T. Mor, P. W. Shor, J. A. Smolin, and B. M. Terhal, Phys. Rev. Lett. 83, 3081 (1999)

[24] D. P. DiVincenzo, T. Mor, P. W. Shor, J. A. Smolin, B. M. Terhal, quant-ph/9908070.

[25] A. Thapliyal, Phys. Rev. A 59 (1999) 3336.

[26] W. Dür, J.I. Cirac, Phys. Rev. A 61 (2000) 042314.

[27] M. Murao, D. Jonathan, M. B. Plenio, V. Vedral, Phys. Rev. A 59 (1999) 156.

[28] D. Janzing and T. Beth, quant-ph/9907042.

[29] D. Aharonov, quant-ph/9910081.

[30] M. Horodecki, P. Horodecki and R. Horodecki, Phys. Rev. Lett. 80, 5239 (1998); P. Horodecki, M. Horodecki and R. Horodecki, ibid. 82, 1046 (1999).

[31] B. M. Terhal, quant-ph/9810091; see also quant-ph/9911057 (to appear in Phys. Lett. A).

[32] A. Jamiołkowski, Rep. Math. Phys. 3, 275 (1972).

[33] S. L. Woronowicz, Rep. Math. Phys. 10, 165 (1976).

[34] K. Życzkowski, P. Horodecki, A. Sanpera, M. Lewenstein Phys. Rev. A 58833 (1998).

[35] B. Terhal, private communication. 